\title{
The Effects of Magnetic Field on Flow Characteristics of High Temperature Conductive Gas in a Cylinder
}

\author{
Mengchun Zhong ${ }^{a}$, Xiaogang Li, Baoquan Mao and Cheng $\mathrm{Li}^{\mathrm{b}}$ \\ Academy of Armored Force Engineering, Beijing 100072, China \\ a969493577@qq.com, blichenyuw@163.com
}

Keywords: Magnetic Field, Plasma, Magneto Hydrodynamics, Propellant Gas, The Lorentz Force.

\begin{abstract}
Considering the conductive properties of the propellant gas and the application potential of the plasma flow control, a magneto hydrodynamic model of the barrel is built and the magneto hydrodynamic control equations are solved by magnetic induction method. The effects of different magnetic fields on flow characteristics of the propellant gas in barrel are numerically studied. The results show that the ways of applied magnetic field and the magnetic field intensity are two main factors influencing the flow anisotropy. The effects of different ways of applied magnetic field on the velocity field of the propellant gas is different, and the corresponding application mode can be selected according to the actual demand. The Lorentz force induced in the plasma can reduce the friction force of the wall and the turbulence intensity of the gas that will result in the decrease of the friction coefficient and the heat transfer from the gas to the wall effectively. When the applied magnetic field is $1 \mathrm{~T}$ and the conductivity is $5000 \mathrm{~s} / \mathrm{m}$, the friction coefficient of the wall decreases about $8.7 \%$.
\end{abstract}

\section{Introduction}

When the temperature is high enough, the material will be difficult to maintain the pure gas state, and its atoms or molecules will ionize leading to the formation of electrons, ions and neutral particle system, known as the plasma. Plasma different from the solid, liquid and gas, is another existence state of materials, also known as the fourth state of substance [1].

Theoretically, the light generated by the combustion of the powder belongs to the violet band, and the part of the light in the violet band is composed of ionized electrons and ions. The experimental measurements also show [2,3] that the propellant containing Cs salt combustion can form about 200 $\mathrm{S} / \mathrm{m}$ conductivity of high temperature gas, and the conductivity of the $1.520 \mathrm{~g} . \mathrm{cm}-3$ high-energy explosives gas containing $\mathrm{Al}$ is up to $18000 \mathrm{~S} / \mathrm{m}$. It can be seen that the process of gunpowder combustion will produce ionized plasma. That is, the gunpowder gas has a certain conductivity.

In the electromagnetic field, the conductive fluid will show some characteristics different from the ordinary flow field. The reasonable use of the interactions between electromagnetic field and the conductive fluid is the key to achieve the flow field control. In the pipe flow control, the research are mainly from two aspects at present $[4,5]$. On the one hand is the flow control in the export of tube, the other hand is the flow control in the elbow.

Considering the conductive properties of the propellant gas and the application potential of the plasma flow control, a magneto hydrodynamic (MHD) model of the barrel is built and the MHD control equations are solved by magnetic induction method in the paper, based on the plasma physics, artillery internal ballistics, advanced gas dynamics, electromagnetic, magnetic fluid dynamics, etc. Finally the effects of different magnetic fields on flow characteristics of the propellant gas in barrel are numerically studied.

\section{MHD Model and Its Solving Method}

MHD description method focuses on the overall behaviors of plasma, which studies the interaction between the conductive fluid and magnetic field macroscopically. MHD method can accurately 
describe the pressure, speed and other macroscopic quantities of the plasma flow field. Therefore, the propellant gas under the magnetic confinement will be treated as magnetic fluid using MHD method to simulate numerically.

\subsection{Basic Assumption.}

Considering the flow of the propellant gas inside the barrel and the characteristics of external magnetic field, make the following basic assumptions:

(1)Regardless of the anisotropy of the electromagnetic medium.

(2)The plasma stream is electrically neutral as a whole, there is no single positive and negative charge.

(3)Regardless of the Hall Effect and ion slip effect.

(4)The plasma stream is a continuous medium.

(5)Only considering the body force generated by the electromagnetic.

\subsection{MHD Control Equation.}

The whole MHD control equations comprehensively consider the interaction between the propellant gas (conductive gas) and the electromagnetic field based on the classical fluid mechanics and electromagnetism. The influence of electromagnetic field on the propellant gas is mainly reflected by adding the source of electromagnetic force and Joule heat on the basis of classical N-S (Navier-Stokes) equation. And the effect of the flow field on the electromagnetic field is mainly reflected by the magnetic induction equation.

MHD mass conservation equation:

$$
\frac{\partial \rho}{\partial t}+\nabla \cdot(\rho U)=0
$$

MHD momentum conservation equation:

$$
\rho \frac{\partial U}{\partial t}=-\nabla p+\rho f_{v}+\nabla \cdot \tau+J \times B
$$

Where $p$ is the pressure, and $f_{v}$ is the body force other than the magnetic force. $\tau$ is the shear stress tensor, and $J \times B$ is the Lorentz force.

MHD energy conservation equation:

$$
\rho \frac{\partial \varepsilon_{f}}{\partial t}=-p \nabla \cdot U+\rho U \cdot f_{v}+U \cdot(\nabla \cdot \tau)+\frac{J^{2}}{\sigma}
$$

Where $\varepsilon_{f}$ is the internal energy of the gas, and $J^{2} / \sigma$ is the Joule heat source item.

The magnetic induction equation mainly includes the Maxwell's equations and the simplified generalized Ohm's law (ignoring the Hall Effect).

Maxwell's equations:

$$
\left\{\begin{array}{l}
J=\frac{1}{\mu_{m}} \nabla \times B \\
\nabla \times E=-\frac{\partial B}{\partial t} \\
\nabla \cdot B=0 \\
Q=J^{2} / \sigma
\end{array}\right.
$$

Where $\mu_{m}$ is the permeability, and $Q$ is the Joule heat.

Ohm's law:

$$
J=\sigma(E+U \times B)
$$

Where $B$ and $E$ are the magnetic and the electric fields, respectively, and $\sigma$ is the electrical conductivity. $J$ is the electric current density vector, and $U$ is the velocity vector of the flow.

\subsection{Solution.}


At present, there are three commonly numerical methods to solve the coupling problem of the conducting fluid and electromagnetic field, namely magnetic induction method, electric potential method and the current method [6]. In the paper, magnetic induction method is used to solve the problem. From Ohm's law and Maxwell's equation, the magnetic induction equation can be derived as:

$$
\frac{\partial b}{\partial t}+(U \cdot \nabla) b=\frac{1}{\mu_{m} \sigma} \nabla^{2} b+(b \cdot \nabla) U
$$

It can be seen that the magnetic induction equation is in the same form as the mass conservation equation, the momentum conservation equation and the energy conservation equation, with the difference being the respective source term. Therefore, they can all be solved by using UDS transport equation solver.

$$
\frac{\partial}{\partial t}(\rho \Phi)+(U \cdot \nabla) \Phi=\nabla \cdot(\Gamma \nabla \Phi)+S(\Phi)
$$

In equation (7), for each term, they represent the transient term, convection term, diffusion term and source term respectively. $\Phi$ is the universal variable, and $\Gamma$ is the diffusion coefficient. $S(\Phi)$ is the corresponding source term for each variable.

\section{Numerical Simulation and Results Analysis}

\subsection{Physical Model and Boundary Condition.}

Considering the characteristics of artillery barrel, the paper chooses the $125 \mathrm{~mm}$ cylinder as the research object. The physical model is shown in Fig.1. The wall's material is the gun steel, and the fluid area is the plasma (partly ionized propellant gas). The entrance is in the yoz plane, and the flow is along $\mathrm{x}$ positive direction.

Considering the characteristics of the propellant gas, the simulation uses the velocity inlet and the pressure outlet. The initial velocity of the plasma stream is $1000 \mathrm{~m} / \mathrm{s}$, the temperature is $3000 \mathrm{~K}$, and the electrical conductivity is $5000 \mathrm{~S} / \mathrm{m}$. The outlet temperature is $1000 \mathrm{~K}$ and the pressure is $0.1 \mathrm{MPa}$. The permeability of the gun steel is $4 \pi \times 10^{7} \mathrm{H} / \mathrm{m}$. The grid is composed of 131989 mesh elements, 407902 mesh faces and 144072 mesh nodes, as shown in Fig.2. The plasma control equation under the magnetic field is coupled solved by the call of MHD module and UDS in FLUENT. Addition to, the second-order upwind scheme is adopted to improve the calculation precision.

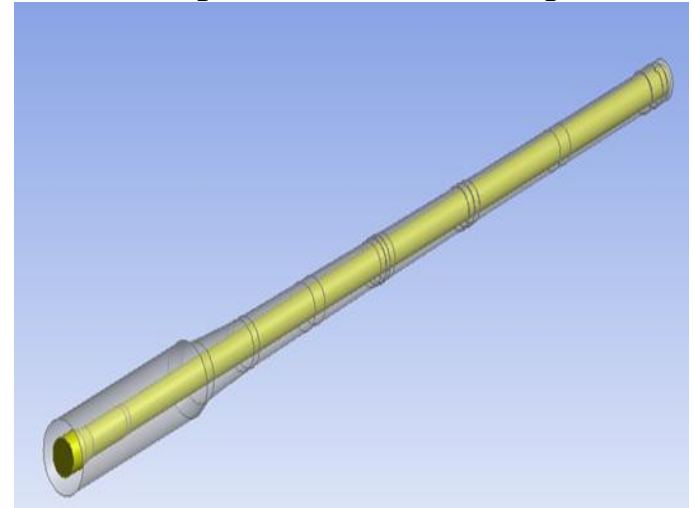

Fig.1 Physical model



Fig.2 Structured grid

\subsection{The Influence of Different Magnetic Field Intensity on the Flow Field in a Circular.}

In order to study the effect of different magnetic field intensity on the flow characteristics of the propellant gas in the tube, a constant magnetic field $B_{\mathrm{Z}}$ with different intensity was applied in the z-axis direction, $0.2 \mathrm{~T}, 0.6 \mathrm{~T}, 1.0 \mathrm{~T}$ respectively.

Fig.3 (a) and (b) show the distributions of the velocity $u$ along the $y$-axis and $z$-axis in the yoz section of the outlet under different magnetic field strengths. Under the applied magnetic field, the velocity $u$ of the plasma decreases obviously, and the amplitude decreases with the increase of the magnetic field. Because in a magnetic environment, the Lorentz force of the plasma is proportional to 
the magnetic field strength, and the direction is the opposite direction of the flow. In the distribution of velocity $u$ along the $z$ axis in Fig. 3 (b), the velocity $u$ when applied magnetic field is slightly larger than the velocity $u$ when there is no magnetic field in the near wall. It becauses the flow also need to meet the continuity conditions of flow while velocity changes.

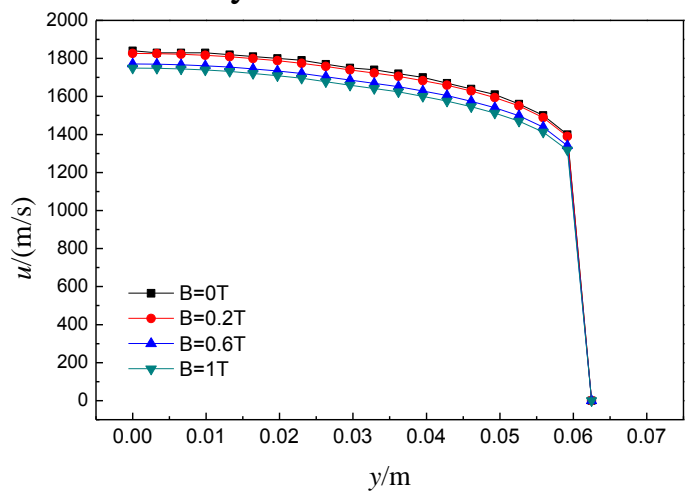

(a)The velocity $u$ along the $y$-axis

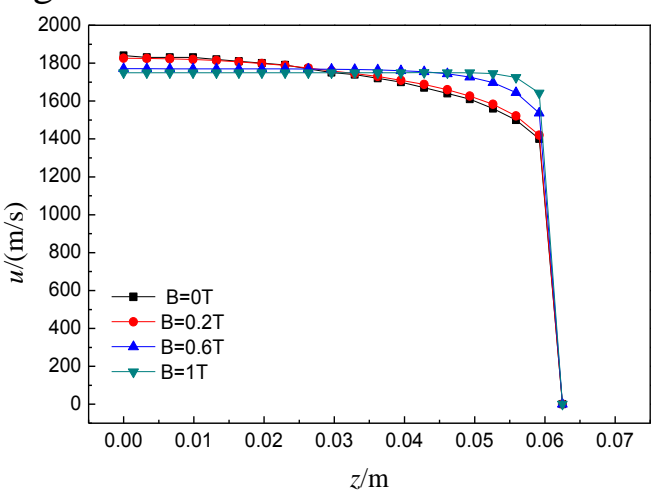

(b)The velocity $\mathrm{u}$ along the $\mathrm{z}$-axis

Fig.3 Variation of velocity $u$ with the intensity of magnetic field

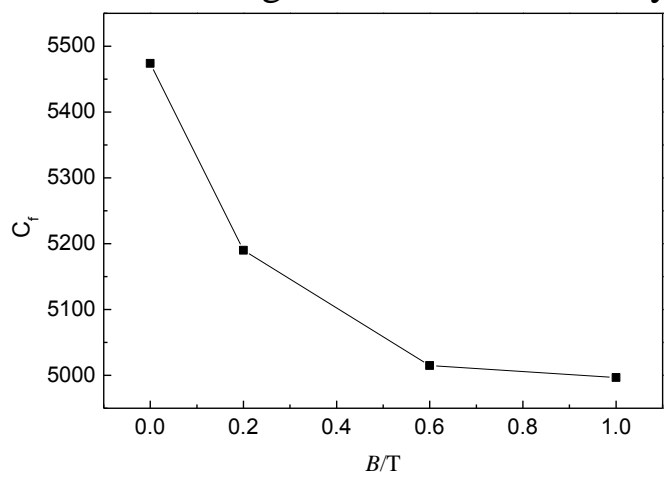

Fig.4 Variation of wall friction coefficient

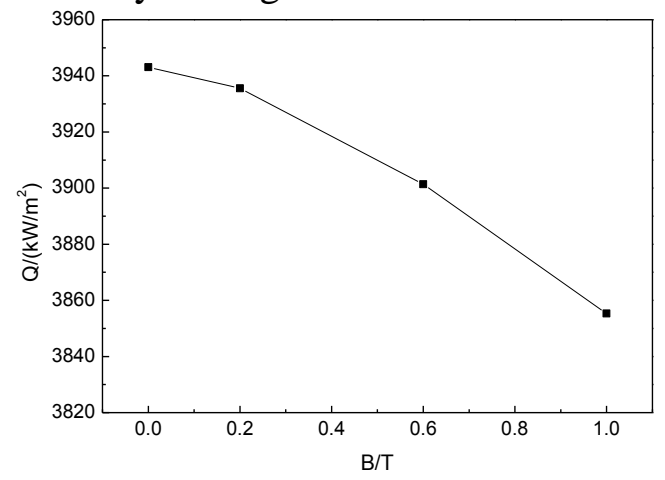

Fig.5 Variation of heat transfer

Fig.4 shows the influence of different magnetic field Intensity on wall friction coefficient $C_{f}$. It can be seen that the friction coefficient of the wall surface decreases obviously, and the decreasing magnitude increases with the increase of the magnetic field intensity. When the applied magnetic field is $1 \mathrm{~T}$, the friction coefficient of the wall decreases about $8.7 \%$. The result agrees well with the theoretical analysis in magnetohydrodynamics.

Figure 5 shows the influence of different magnetic field intensity on heat transfer. It can be seen that the heat transfer of the plasma to the wall surface is obviously decreased with the increase of the magnetic field intensity. Because of the existence of the Lorentz force, the plasma density near the wall is larger than that at the center of the tube. That is, a layer of plasma is formed at the near wall, and the presence of the magnetic field reduces the turbulence intensity of the plasma near the wall. From the fluid mechanics and heat transfer theory, it can be seen that the capacity of heat transfer of the flow and the friction will decrease, which will eventually reduce the heat transfer to the outer wall.

\subsection{The effect of the different ways of applied magnetic field on the characteristics of the flow}

field.

In order to study the effect of different magnetic fields on the characteristics of the flow field in the barrel, four methods of applied magnetic field are proposed: uniform one-dimensional axial magnetic field (along the $x$-axis direction), uniform one-dimensional longitudinal magnetic field (along the $y$-axis direction), uniform one-dimensional transverse magnetic field (along the $z$-axis direction), and uniform two-dimensional magnetic field, as shown in Fig.6. 


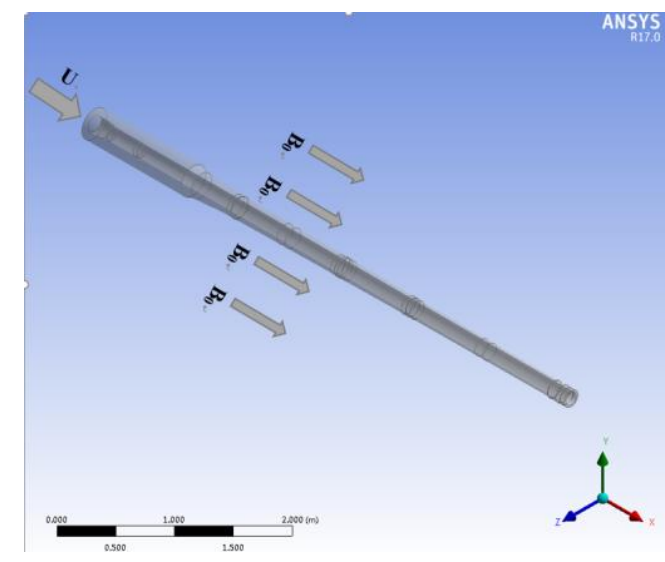

(a) one-dimensional axial magnetic field

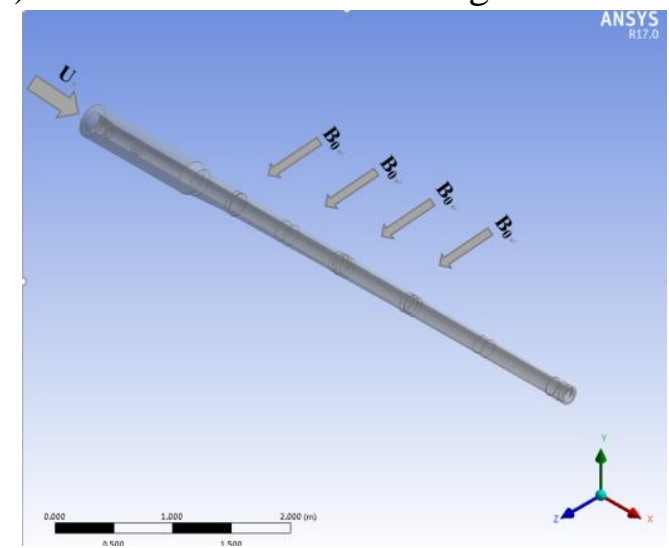

(c) one-dimensional transverse magnetic field

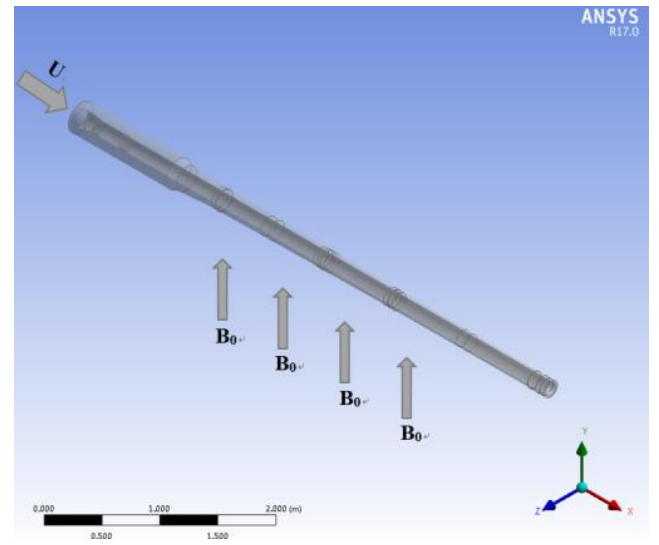

(b) one-dimensional longitudinal magnetic field

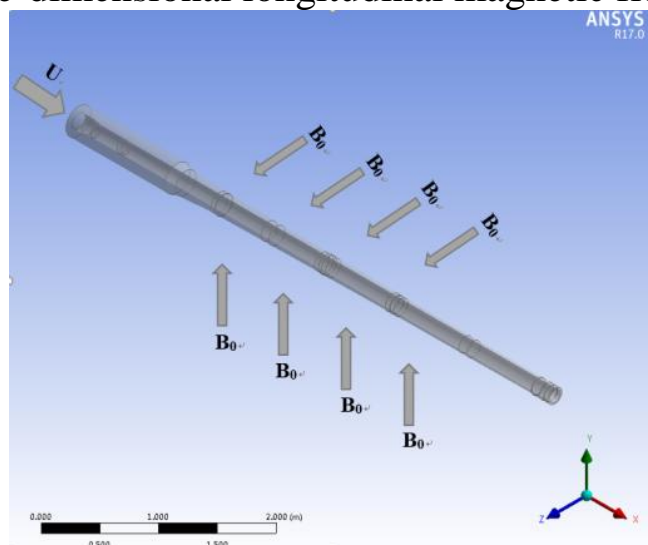

(d) two-dimensional magnetic field

Fig.6 different ways of applied magnetic field

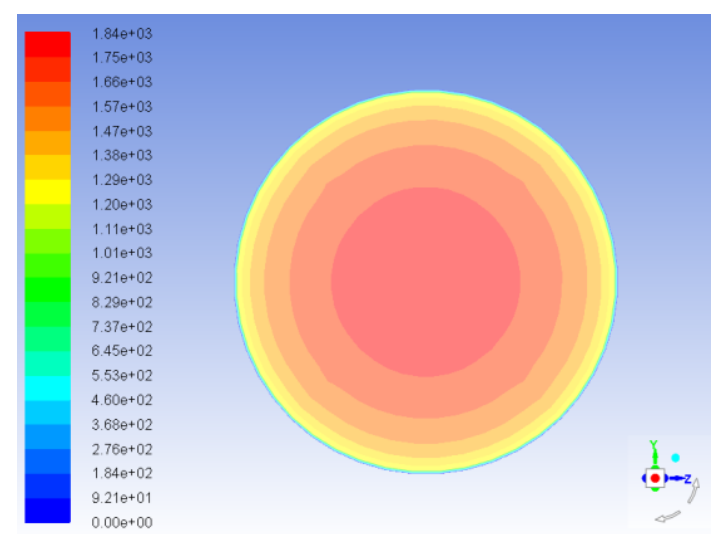

(a) one-dimensional axial magnetic field

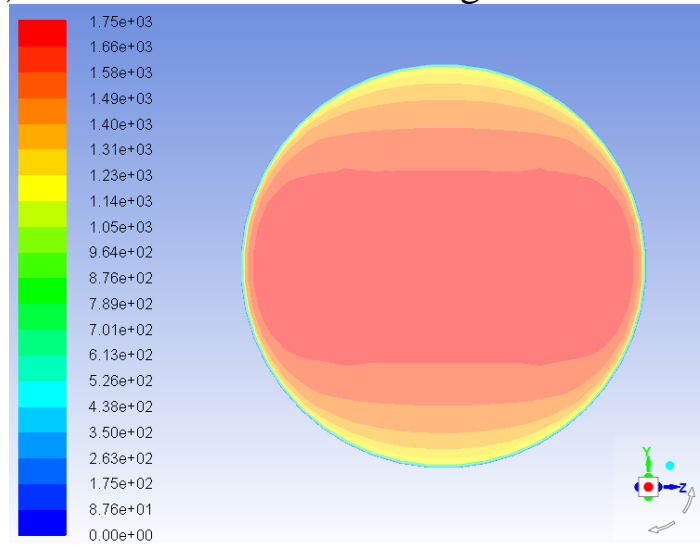

(c) one-dimensional transverse magnetic field

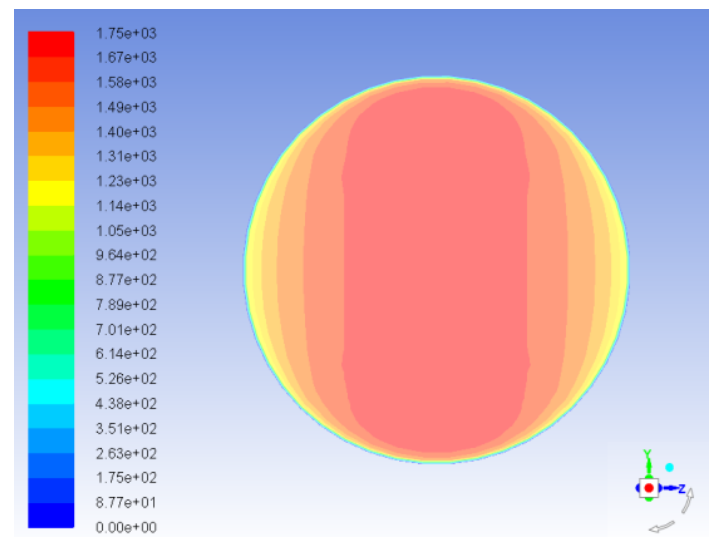

(b) one-dimensional longitudinal magnetic field

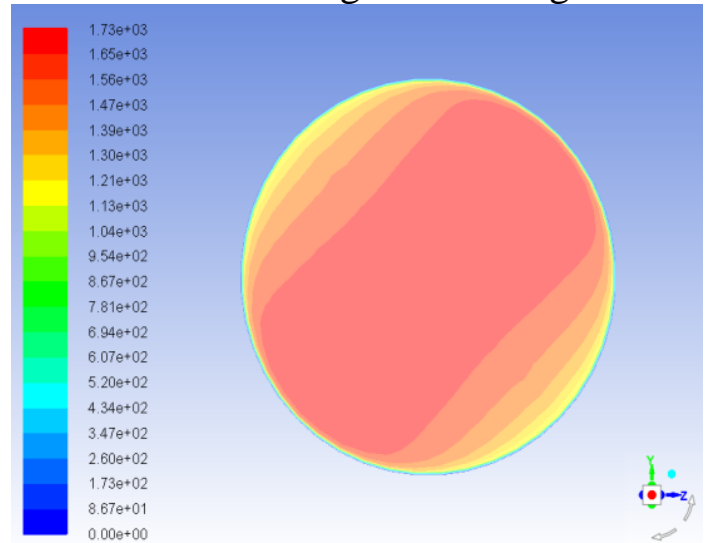

(d) two-dimensional magnetic field Fig.7 The distribution of the velocity $u$ on the outlet section 
Fig.7 (a), (b), (c) and (d) show the distribution of the velocity $u$ on the yoz cross section under different magnetic fields. It can be seen, in addition to the application of one-dimensional uniform axial magnetic field, the velocity $u$ on the outlet section are anisotropic in the other three cases. Under the uniform one-dimensional longitudinal magnetic field, the velocity along the $y$-axis is greater than the velocity along the $z$-axis, and under the uniform one-dimensional transverse magnetic field, the velocity along the $z$-axis is greater than the velocity along the $y$-axis. Under the uniform two-dimensional magnetic field, the velocity along the clockwise rotation $45^{\circ}$ direction is greater than the other direction. Since the pulsating velocity $U$ is considered, the resulting current density expression is $J=\sigma(E+U \times B)$. The Lorentz force induced by interacts between the current and the magnetic field is:

$$
F=J \times B=\sigma[E \times B-(B \times B) U+(U \times B) B]
$$

If the velocity is perpendicular to the direction of the magnetic field, the direction of the Lorentz force is in the opposite direction to the pulsating velocity component since the latter term is zero. While the direction of the velocity is the same as the magnetic field, the Lorentz force will be zero due to the existence of the latter term. The above equation shows that the pulsation perpendicular to the direction of the magnetic field will be inhibited, while the pulsation parallel to the direction of the magnetic field is unaffected. Therefore, the flow is anisotropic in the cross section.

\section{Summary}

A MHD model of the barrel is built and the MHD control equations are solved by magnetic induction method in the paper. Finally the effects of different magnetic fields on flow characteristics of the propellant gas in barrel are numerically studied. The conclusions are as follows:

(1) Under the applied magnetic field, the current is induced in the plasma, and the induced Lorentz force impedes the fluid flow to a certain extent and decreases the velocity. At the same time, because of the different Lorentz forces along the $y$-axis and $z$-axis, the flow of propellant gas shows anisotropy.

(2) The friction coefficient and the shearing stress of the wall are reduced by the Lorentz force, which decreases the friction loss between the plasma and the wall. When the applied magnetic field is $1 \mathrm{~T}$, the friction coefficient of the wall decreases about $8.7 \%$.

(3) Because of the existence of the Lorentz force, a layer of high-density plasma (sheath) gather near the wall, while the existence of magnetic field can restrain the turbulence intensity of the plasma. Eventually the capacity of heat transfer will reduce correspondly.

(4) The effects of different ways of applied magnetic field on the velocity field of the propellant gas is different, and the corresponding application mode can be selected according to the actual demand.

\section{References}

[1] ZHENG Chunkai. Plasma physics [M]. Beijing: Peking University Press, 2012.

[2] ZHOU Lin, LIAO Yingqiang, XU Gengguang. Experimental measurement of electrical conductivity of detonation products [J]. Energetic Materials, 2005, 13(3):148-153.

[3] ZHOU Lin, XIE Zhongyuan, SHAO Qinxing. Study on the electrical conductivity of combustion products containing Cs salt [J]. Energetic Materials, 2012, 17(1):100-105.

[4] Sinha A, Alkandry H, Kearney-Fischer M, et al. The impulse response of a high-speed jet forced with localized arc filament plasma actuators [J]. Physics of Fluids,2012, 24(12): 125104.

[5] Speth R, Gaitonde D V. Parametric study of a Mach 1.3 cold jet excited by the flapping mode using plasma actuators [J]. Computers \& Fluids, 2013, 84: 16-34.

[6] Fan Yuehua, Jiang Chongwen, Gao Zhenxun, et al. Review of the magnetohydrodynamic turbulence and its numerical simulation. Mechanics in Engineering, 2016, 38(1):14-21. 